\title{
Quantification of Cell Traction Force of Osteoblast Cells Using Si Nanopillar-Based Mechanical Sensor
}

\author{
Yiming Jin ${ }^{\dagger}$, Yalan Zhang ${ }^{1, \dagger}$, Han Ouyang, \\ Mingzeng Peng, Junyi Zhai* and Zhou Li** \\ Beijing Institute of Nanoenergy and Nanosystems, \\ Chinese Academy of Sciences, Beijing 100083, China \\ ${ }^{1}$ School of Biological Science and Medical Engineering, \\ Beijing University of Aeronautics and Astronautics, Beijing 100083, China
}

(Received May 7, 2015; accepted July 21, 2015)

Key words: silicon, nanopillar, cell traction force, osteoblasts, finite element analysis

A large number of methods have been developed to quantify the cell traction force and its distribution owing to its unique significance in the field of biocytology and tissue engineering. Silicon nanopillar arrays are excellent materials for measuring the force and preventing the problems of other methods because of their excellent mechanical strength and spatial resolution. In this study, silicon nanopillar arrays were prepared by laser interference lithography, and nanopillars were bent by cells after culture for a certain period of time because cells started to migrate along a certain direction. Finite element analysis was conducted to simulate the relationship between the cell traction force and the corresponding lateral displacement. We found that the displacement is positively associated with the transverse force, the average traction force of one single osteoblast measured by Si nanopillars was $8.5 \mu \mathrm{N}$ and the maxima was $10.8 \mu \mathrm{N}$.

\section{Introduction}

The traction force is one of the most important properties of cells; it involves many sophisticated biological signal transduction channels and plays a significant role in the proliferation, differentiation, apoptosis, migration and contraction of cells. What's more, cell traction force is highly linked to the occurrence and development of many diseases such as tumors. ${ }^{(1)}$ Thus, it is of great physiological and pathological significance to conduct some quantitative research studies on the characteristics of cell activities at a single cell level, ${ }^{(2-4)}$ which may provide a new cellular level diagnostic technique clinically in the future.

${ }^{*}$ Corresponding author: e-mail: jyzhai@binn.cas.cn

${ }^{* *}$ Corresponding author: e-mail: zli@binn.cas.cn

†These authors contributed equally to this work. 
A variety of methods, such as the use of cell-populated collagen gel, ${ }^{(5,6)}$ thin silicone membranes, ${ }^{(7)}$ and cell traction force microscopy, ${ }^{(8)}$ have been applied to quantify the cell traction force. A widely adopted improved method is the use of a micropost force sensor array; ${ }^{(9)}$ it enables the measurement of the traction force of one single cell. However, the following problems still exist: A polymer-based micropost always exhibits the selfelongation effect, that is, the micropost not only bends but also stretches along the axis direction. The resolution of a soft substrate is not as good as that of a hard one; thus, it contributes to a larger space between microposts; namely, at a far smaller number of microposts, cells may fall off under this circumstance. ${ }^{(10)}$

Silicon is a widely used semiconductor material, and the fabrication of silicon nanostructures is central to many processes in a variety of fields and applications, including sensing, ${ }^{(11,12)}$ photovoltaics, ${ }^{(13)}$ and energy storage. ${ }^{(14)}$ To overcome those problems mentioned above, we adopted Si nanopillars as the sensors to quantify the cell traction force because of their well-known properties and mature fabrication technologies. Silicon nanopillars have been demonstrated as unique probes for quantifying biological processes at a high spatial resolution. ${ }^{(15,16)}$ In addition, the high Young's modulus and aspect ratio of Si nanopillars guarantee their mechanical strength so that they bend under lateral force without stretching; thus, the obtained results would be more precise and reliable. Figure 1 depicts that the Si nanopillars are vertical when the cells attach to the Si nanopillars for a short period of time, but a couple of hours later after the cells extend thoroughly and start to migrate, it is observed that the Si nanopillars are bent by cells owing to the traction force caused by the migration of cells.

\section{Materials and Methods}

\subsection{Preparation of Si nanopillars}

The fabrication process for Si nanopillar arrays is shown in detail in Fig. 2. A Si wafer was cleaned by rinsing with acetone, ethanol, and deionized water for several times, and then spin-coated with a photoresist. Laser interference lithography (LIL) ${ }^{(17,18)}$ was used to form arrays of photoresist posts. Then, $\mathrm{Ag}$ was deposited on the substrate by the magnetron sputtering system acting as a catalyst. After etching in the mixture

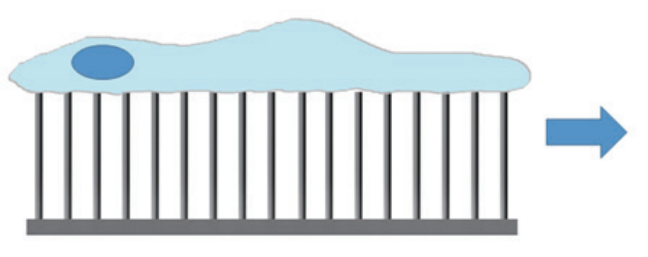

(a)

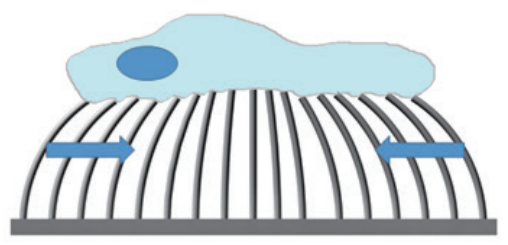

(b)

Fig. 1. (Color online) Schematic diagrams to illustrate (a) that the Si nanopillars are vertical first, and (b) that the Si nanopillars are bent by cells after culture for a period of time. 


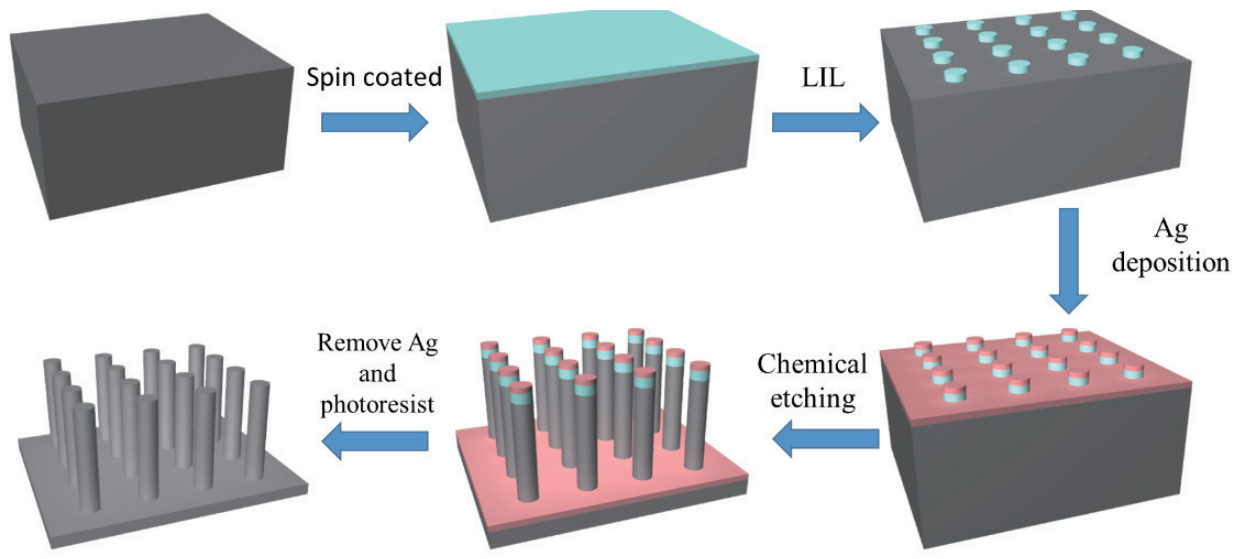

Fig. 2. (Color online) Schematics of the fabrication process for Si nanopillar arrays.

solution of $\mathrm{H}_{2} \mathrm{O}$ and $\mathrm{HF}$ for several minutes, the $\mathrm{Ag}$ and photoresist were removed from the tip of arrays, and finally uniform Si nanopillar arrays were obtained.

The obtained neatly arranged nanopillars were $2 \mu \mathrm{m}$ in height, and the diameters of the upper and middle parts of the nanopillars are 250 and $450 \mathrm{~nm}$, respectively, as presented in Fig. 3(a). To optimize the bending effect of the nanopillars and obtain more precise results, the aspect ratios of the nanopillars were increased by reoxidation and chemical etching. The Si nanopillars were put into a muffle furnace and reoxidized at $850{ }^{\circ} \mathrm{C}$ for $80 \mathrm{~min}$, then immersed in the etching solution containing the mixture of $\mathrm{H}_{2} \mathrm{O}$ and $40 \% \mathrm{HF}$ for $10 \mathrm{~min}$. The sizes of the etched nanopillars decreased evidently, with $180 \mathrm{~nm}$ at the top and $250 \mathrm{~nm}$ in the middle, as depicted in Fig. 3(b).

\subsection{Culture of osteoblasts cells}

MC3T3-E1 osteoblasts were used in this study. They were maintained in Dulbecco's modified Eagle's medium (DMEM) supplemented with 10\% fetal bovine serum (FBS, Hyclone) and $1 \%$ penicillin/streptomycin (Gibco) at $37^{\circ} \mathrm{C}$ in a humidified atmosphere of $5 \% \mathrm{CO}_{2}$.

Suspended cells were prepared from anchorage cells cultured in Petri dishes for $3 \mathrm{~d}$. The aligned Si nanopillar arrays were sterilized by immersion in $75 \%$ medical standard ethanol for $2 \mathrm{~h}$ and exposure to a high-dose UV light for another $2 \mathrm{~h}$. After sterilization, the aligned Si nanopillar arrays were rinsed in deionized (DI) water 3 times and dried using extra pure nitrogen gas. The aligned Si nanopillar arrays were immersed in the culture medium in Petri dishes prior to adding the cells. Suspended MC3T3-E1 cells were seeded at a density of $1 \times 10^{5}$ cells $/ \mathrm{ml}$ onto Si nanopillar arrays and shaken slightly for further dispersion, then the cells were incubated at $37^{\circ} \mathrm{C}$ in $5 \% \mathrm{CO}_{2}$.

To obtain the contraction force of a single cell, the aligned Si nanopillar arrays were taken out of the cell culture medium at $96 \mathrm{~h}$ before confluence and the cells attached 


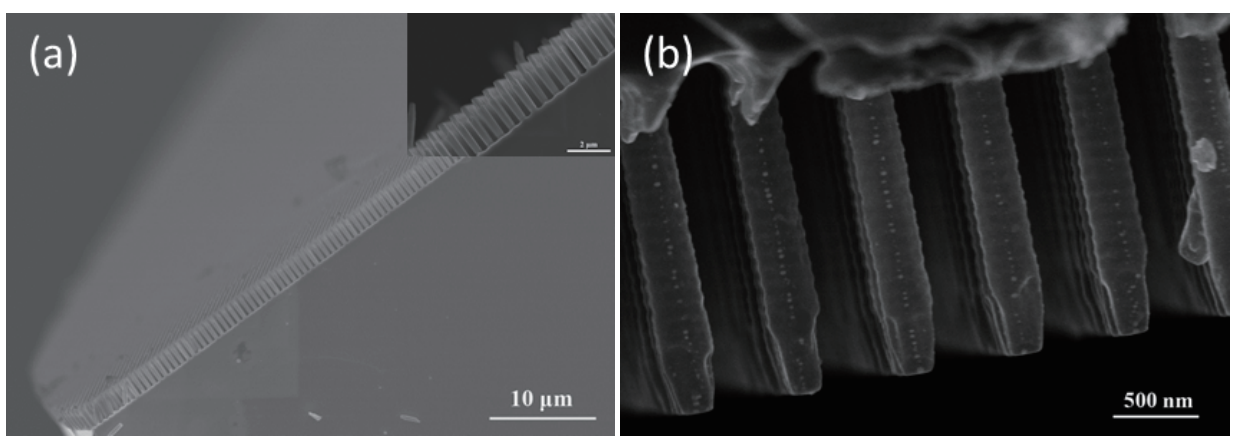

Fig. 3. Cross-sectional SEM images of (a) Si nanopillar arrays prepared by laser interference lithography [the inset of (a) is a high-magnification image that shows the details] and (b) $\mathrm{Si}$ nanopillar arrays after chemical etching with the mixture solution of $\mathrm{H}_{2} \mathrm{O}$ and $\mathrm{HF}$.

to them were fixed immediately. The samples were fixed with $2.5 \%$ glutaraldehyde and $4 \%$ paraformaldehyde (in $1 \times$ PBS) for $2 \mathrm{~h}$, maintaining the morphology, size and essential state of the cells for observation and measurement. Subsequently, the samples were further washed three times with PBS buffer. After immersing the samples in $1 \%$ chlorauric acid, the samples were dehydrated by increasing the concentration of ethanol and air-dried.

\section{Results and Discussion}

It can be seen from Fig. 4(a) that osteoblasts cells grew fairly well on Si nanopillar arrays; the inset is a higher magnification image, which demonstrates the state and morphology of cells. Figure 4(b) shows a scanning electron microscopy (SEM) image of one single cell, and it is clearly seen from Fig. 4(c) that the nanopillars underneath are bent by the cell as a result of cell traction force.

To quantify the tractrion force of one single cell, we adopted a simulation software COMSOL package to carry out finite element analysis to determine the relationship between the lateral force applied by cells and the corresponding displacement of nanopillars. The geometry of the setting model is a truncated cone, the height is $2 \mu \mathrm{m}$, and the diameters of the bottom and top surfaces are 250 and $180 \mathrm{~nm}$, respectively. The basic mechanical properties of the model material refer to the values given by the previous literature: the Young's modulus $E$ is $150 \mathrm{GPa}$, the Poisson's ratio $v$ is 0.278 , and the density $\rho$ equals to $2330 \mathrm{~kg} / \mathrm{m}^{3} .^{(10)}$ The bottom surface is set as a fixed constraint and the modeled deflection force applied to the top surface is paralle to the substrate. The insets of Figs. 5(a) and 5(b) show the images of Si nanopillars bent by the cell traction force, which correspond to 9.1 and $4.1 \mu \mathrm{N}$, respectively, as simulated by COMSOL; the greater the cell traction force, the larger the bending extent. The video in the supporting 

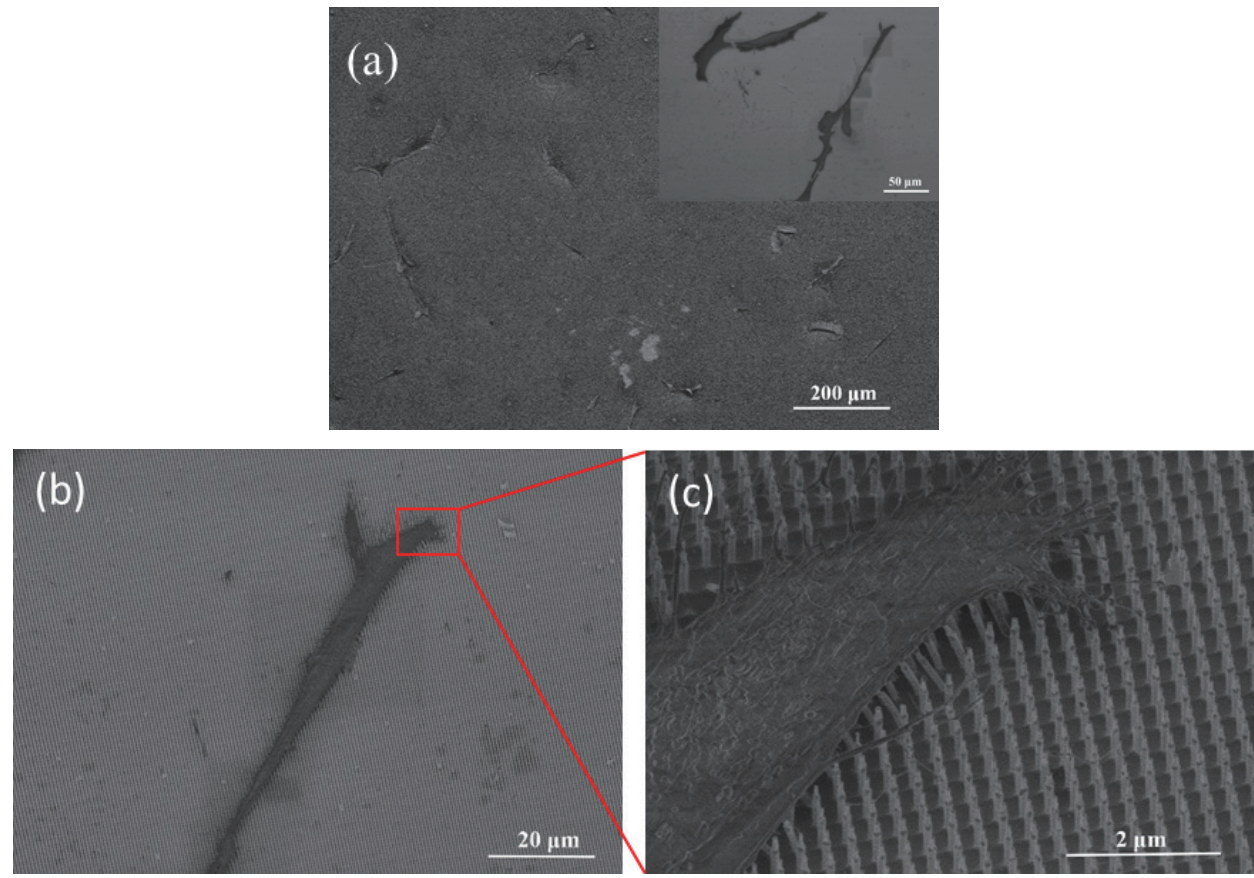

Fig. 4. (Color online) (a) Overview of the cells cultured on Si nanopillar arrays. The inset is an enlarged section. (b) Bird's-eye view of one single osteoblast cell and (c) the bent Si nanopillars underneath.

$F=9.1 \mathrm{uN} \quad X$-direction deflection $(\mathrm{nm})$

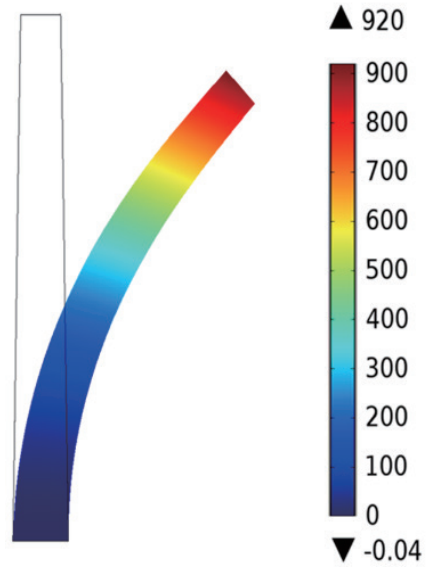

(a)

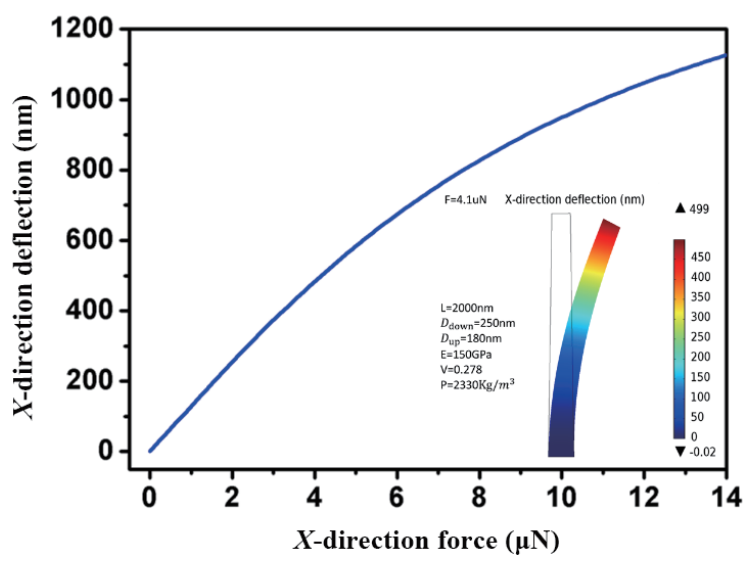

(b)

Fig. 5. (Color online) (a) Bending morphology of Si nanopillars under $9.1 \mu \mathrm{N}$ lateral force and (b) relationship between the applied force parallel to the substrate and the tip deflection distance of a Si nanopillar calculated via finite element analysis. The inset is the bending morphology of $\mathrm{Si}$ nanopillars under $4.1 \mu \mathrm{N}$ lateral force and corresponding parameters. 

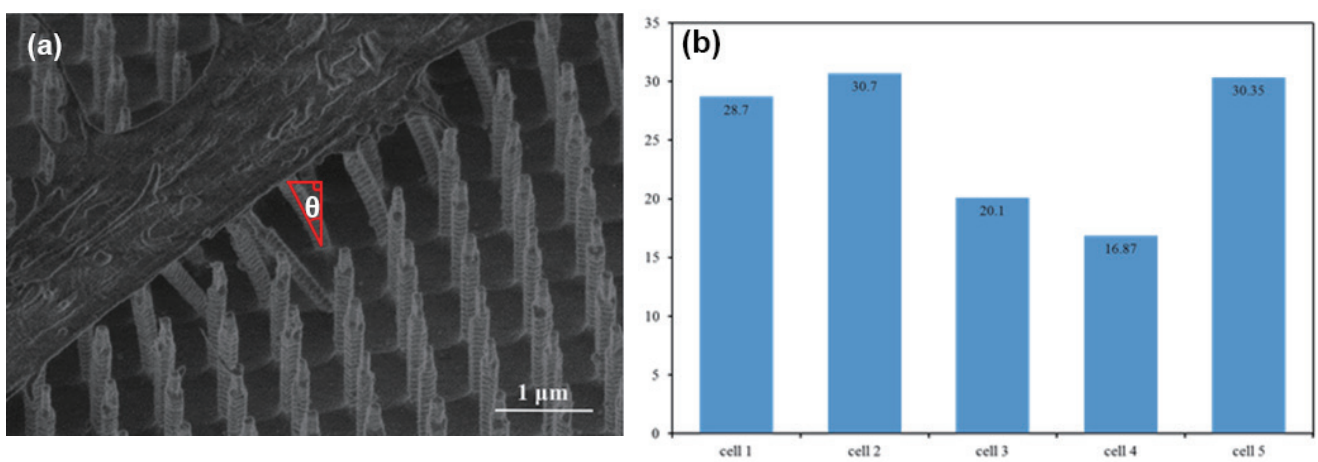

Fig. 6. (Color online) (a) Schematic diagram that describes the bend angles applied in the determination of cell traction force. average bend angles of different cells.

(b) The statistical histogram demonstrates the obtained

information is provided to illustrate the bending morphology of Si nanopillars as cell traction force increases. Figure 5(a) also proves that Si nanopillars are suitable materials for measuring the force because they maintain excellent mechanical strength even when bent at a rather large angle. Figure 5(b) shows that the lateral displacement is positively associated with the cell traction force, and that the obtained curve could be used for calibration to quantify the transverse force from the measured lateral displacement of the nanopillar tip.

To guage the actual cell traction force of osteoblasts, we took many SEM images of nanopillars bent by cells and analyzed the corresponding bend angles. In this case, the bend angle is defined as the angle between the vertical beam direction and the line of the top and bottom surface centers, as described in Fig. 6(a). We measured the bend angles of Si nanopillars in different areas from cells 1 to 5 , and then further computed the average bend angle of every cell as depicted in Fig. 6(b). From all the data we obtained, the calculated average bend angle is $25.54^{\circ}$, so the average lateral displacement is $2 \mu \mathrm{m}$ $\times \sin \left(25.54^{\circ}\right)=0.86 \mu \mathrm{m}$, and from Fig. $5(\mathrm{c})$, the average cell traction force of one single osteoblast is approximately $8.5 \mu \mathrm{N}$.

\section{Conclusions}

We propose a method of quantifying the cell traction force of osteoblasts. COMSOL is used to simulate the relationship between the cell traction force and the lateral displacement of the model material, which is determined by measuring the corresponding bend angle. A Si nanopillar array is an excellent material used in the field of cell traction force quantification because of its excellent mechanical strength and high spatial resolution. The use of sensors based on Si nanopillar arrays is indeed convenient and reliable for quantifying the cell traction force. 


\section{Acknowledgements}

The work was supported by NSFC 31200702, Beijing Municipal NSF 713212 and the "Thousands Talents" program for a pioneer researcher and his innovation team, Beijing Nova Program Z121103002512019 and Beijing Municipal Science \& Technology Commission Z131100006013004.

\section{References}

1 Y. L. Wu, X. D. Chen, J. Z. Zeng and X. K. Zhang: China Patent No. 104359876A (2014).

2 E. Mussig, T. Steinberg, S. Schulz, J. P. Spatz, J. Ulmer, N. Grabe, A. Kohl, G. Komposch and P. Tomakidi: Adv. Funct. Mater. 18 (2008) 2919.

3 N. Q. Balaban, U. S. Schwarz, D. Riveline, P. Goichberg, G. Tzur, I. Sabanay, D. Mahalu, S. Safran, A. Bershadsky and L. Addadi: Nat. Cell Biol. 3 (2001) 466.

4 N. J. Sniadecki, R. A. Desai, S. A. Ruiz and C. S. Chen: Ann. Biomed. Eng. 34 (2006) 59.

5 B. H. Campbell, W. W. Clark and J. H. Wang: J. Biomech. 36 (2003) 137.

6 C. M. Lo, H.B. Wang, M. Dembo and Y. L. Wang: Biophys. J. 79 (2000) 144.

7 A. K. Harris, D. Stopak and P. Wild: Nature 290 (1981) 249.

8 J. P. Butler, I. M. Tolic-Norrelykke, B. Fabry and J. J. Fredberg: Am. J. Physiol.: Cell Physiol. 282 (2002) 595.

9 J. H. C. Wang and J. S. Lin: Biomech. Model. Mechanobiol. 6 (2007) 361.

10 Z. Li, J. H. Song, G. Mantini, M. Y. Lu, H. Fang, C. Falconi and Z. L. Wang: Nano Lett. 9 (2009) 3575.

11 H. C. Jeon, C. J. Heo, S. Y. Lee and S. M. Yang: Adv. Funct. Mater. 22 (2012) 4268.

12 H. D. Fard, S. Khatami and N. Izadi: Sens. Mater. 25 (2013) 297.

13 X. L. Li: Curr. Opin. Solid State Mater. Sci. 16 (2012) 71.

14 C. C. Lin, Y. C. Yen, H. C. Wu and N. L. Wu: J. Chin. Chem. Soc. 59 (2012) 1226

15 F. Patolsky, B. P. Timko, G. Yu, Y. Fang, A. B. Greytak, G. Zheng and C. M. Lieber: Science 313 (2006) 5790.

16 C. M. Lieber and Z. L. Wang: MRS Bull. 32 (2007) 99.

17 J. Boor, N. Geyer, J. V. Wittemann, U. Gösele and V. Schmidt: Nanotechnology 21 (2010) 095302 .

18 W. K. Choi, T. H. Liew and M. K. Dawood: Nano Lett. 8 (2008) 3799. 